EESTI NSV TEADUSTE AKADEEMIA TOIMETISED. $X$ KOIDE

FOOSIKALIS-MATEMAATILISTE JA TEHNILISTE TEADUSTE SEERIA. 1961, NR. 4

ИЗВЕСТИЯ АКАДЕМИИ НАУК ЭСТОНСКОИ ССР ТОМ $X$

СЕРИЯ ФИЗИКО-МАТЕМАТИЧЕСКИХ И ТЕХНИЧЕСКИХ НАУК. 1961. ㄴ. 4

\title{
ИНДУКТИВНОЕ СОПРОТИВЛЕНИЕ, ОБУСЛОВЛЕННОЕ ОСНОВНЫМ МАГНИТНЫМ ПОЛЕМ В ЛИНЕИНОМ ЦИЛИНДРИЧЕСКОМ НАСОСЕ
}

\section{P. IIAPTC}

В настоящее время количество работ, посвященных теории цилиндрического насоса, не велико [']. В данной статье дается вывод индуктивного сопротивления $X_{m}$, обусловленного основным магнитным полем.

Согласно поставленной задачи ниже рассматривается распределение магнитного поля в канале и во внутреннем статоре при отсутствии токов в канале (фиг. 1).

Из условия непрерывности силовых линий любой $v$-ой пространственной гармонической магнитного поля в $n$-ом слое $\operatorname{div} \bar{B}_{v n}=0$ получается

$$
\frac{1}{R} \frac{\partial}{\partial R}\left(R B_{R v n}\right)+\frac{\partial B_{z v n}}{\partial z}=0
$$

при допущении, что поле от угловой координаты не зависит. В уравнении (i)_приняты обозначения:

$R, z-$ координаты в цилиндрической системе көөрдинат; $B_{R v n}$ - радиальная составляющая $v$-ой пространственной гармонической магнитной индукции в $n$-ом слое; $B_{z v n}-$ аксиальная соетавляющая $\boldsymbol{v}$-ой пространственной гармонической магнитной индукции в n-ом слое.

При допущении постоянства радиальной составляющей магннтной проницаемости $\mu_{R n}$ и аксиальной составляющей магнитной проницаемости $\mu_{z n}$ в n-ом слое составляющие магнитной индукции могут быть выражены через соответствующие составляющие (радиальные и аксиальные) $v$-ой пространственной гармонической в n-ом слое напряженности магнитного поля, т. е.

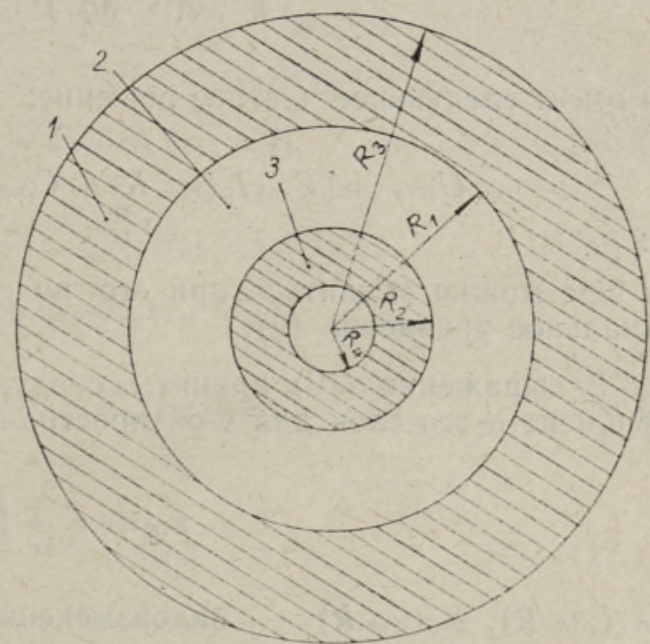

Фиг. 1. Поперечный разрез идеализированного цилиндрического насоса: 1 - внешний статор; 2 - канал; 3 - внутренний статор. Обмотка расположена в пазах внешнего статора. 


$$
\begin{aligned}
& B_{R v n}=\mu_{R n} H_{R v n}, \\
& B_{z \vee n}=\mu_{z n} H_{z \vee n} .
\end{aligned}
$$

В цилиндрическом насосе для определения $X_{m}$ неббходимо рассматривать два слоя: во-первых — воздушный зазор и, во-вторых - внутренний статор.

ПЈскольку на практике встречаются задачи по определению составляющих магнитного поля в слоях с различными магнитными проницаемостями (обработка внутренней поверхности труб и т. д.), в дальнейшем в целях большей общности получаемого решения учитьіваются различные составляющие магнитной проницаемости.

При подстановке (2) и (3) в выражение (1) после элементарного преобразовання получается

$$
\frac{1}{R} \frac{\partial}{\partial R}\left(R H_{R v n}\right)+\frac{\mu_{z n}}{\mu_{R n}} \frac{\partial H_{z v n}}{\partial z}=0
$$

Пользуясь понятием о скалярном потенциале $v$-ой пространственной гармонической магнитного поля в $n$-ом слое, отдельные составляющие напряженности магнитного поля могут быть определены следующим образом:

$$
\begin{gathered}
H_{R v n}=-\frac{\partial U_{H \vee n}}{\partial z}, \\
H_{z \vee n}=-\frac{\partial U_{H \vee n}}{\partial z}
\end{gathered}
$$

На основании (5) и (6) уравнение (4) приннмает вид

$$
\frac{1}{R} \frac{\partial}{\partial R}\left(R \frac{\partial U_{H v n}}{\partial R}\right)+\frac{\mu_{z n}}{\mu_{R n}} \frac{\partial^{2} U_{H v n}}{\partial z^{2}}=0
$$

и инеет следуюшее частное решение:

$$
U_{H v n}=\left[C_{v n 1} I_{0}\left(x_{v n} R\right)+C_{v n 2} K_{0}\left(x_{v n} R\right)\right] \cos v \frac{\pi}{\tau_{1}} z,
$$

в чем можно убедиться прн его подстановке в исходное дифференциальное уравнение (9).

В выражении (10) приняты следующие обозначения: $\varkappa_{v n}-$ коэффициент $n$-ого слоя для $v$-ой пространственной гармонической, равный

$$
x_{v n}=v \frac{\pi}{\tau_{1}} \sqrt{\frac{\mu_{z n}}{\mu_{R n}}}
$$

$I_{0}\left(\varkappa_{v n} R\right), K_{0}\left(\varkappa_{v n} R\right)$ - видоизмененные функции Бесселя нулевого лорядка соответственно первого и второго рода аргумента $\boldsymbol{x}_{v n} R$; $C_{\text {vn1 }}, C_{\text {v } 2}$ - первая и вторая постоянная интегрирования для $v$-ой пространственной гармонической в $n$-ом слое; $\tau_{1}$ - полюсное деление.

Пользуясь выражениями $(10),(5),(6)$ и (2), получаем 


$$
\begin{aligned}
& H_{z v n}=v \frac{\pi}{\tau_{1}}\left[C_{v n 1} I_{0}\left(\varkappa_{v n} R\right)+C_{v n 2} K_{0}\left(\varkappa_{v n} R\right)\right] \sin v \frac{\pi}{\tau_{1}} z, \\
& B_{R v n}=-\mu_{R n} \varkappa_{v n}\left[C_{v n 1} I_{1}\left(\varkappa_{v n} R\right)-C_{v n 2} K_{1}\left(\varkappa_{v n} R\right)\right] \cos v \frac{\pi}{\tau_{1}} z,
\end{aligned}
$$

так как

$$
\begin{aligned}
& I_{0}^{\prime}=I_{1}, \\
& K_{0}^{\prime}=-K_{1} .
\end{aligned}
$$

Для определения постоянных интегрирования можно пользоваться следующими граничными условиями:

$$
\text { 1. При } R=R_{1} \quad B_{R^{v 1} 1}=B_{\text {\% }} \cos v \frac{\pi}{\tau_{1}} z \text {, }
$$

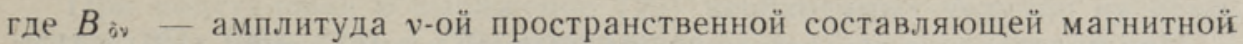
индукции на поверхности статора,

$$
\begin{array}{lll}
\text { 2. При } & R=R_{2} & B_{R v 1}=B_{R v 2}, \\
\text { 3. При } & R=R_{4} & B_{R v 2}=0,
\end{array}
$$

откуда следует, что проникновением магнитного потока в полость внутреннего статора, изготовленного из электротехнической листовой стали, пренебрегают,

4. При $R=R_{2} \quad H_{z \vee 1}=H_{2 \times 2}$.

На основании (15.1)-(15.4) и, пользуясь общими выражениями (12) и (13), получена следующая система уравнений для первого и второго слоя:

$$
\begin{gathered}
-\mu_{R 1} \varkappa_{v 1}\left[C_{v 11} I_{1}\left(\varkappa_{v 1} R_{1}\right)-C_{v 12} K_{1}\left(\varkappa_{v 1} R_{1}\right)\right]=B_{\nu v} \\
\mu_{R 1} \varkappa_{v 1}\left[C_{v 11} I_{1}\left(\varkappa_{v 1} R_{2}\right)-C_{v 12} K_{1}\left(\varkappa_{v 1} R_{2}\right)\right]= \\
=\mu_{R 2} \varkappa_{v 2}\left[C_{v 21} I_{1}\left(\varkappa_{v 2} R_{2}\right)-C_{v 22} K_{1}\left(\varkappa_{v 2} R_{2}\right)\right] \\
C_{v 21} I_{1}\left(\varkappa_{v 2} R_{4}\right)-C_{v 22} K_{1}\left(\varkappa_{v 2} R_{4}\right)=0 \\
C_{v 11} I_{0}\left(\varkappa_{v 1} R_{2}\right)+C_{v 12} K_{0}\left(\varkappa_{v 1} R_{2}\right)= \\
=C_{v 21} I_{0}\left(\varkappa_{v 2} R_{2}\right)+C_{v 22} K_{0}\left(\varkappa_{v 2} R_{2}\right)
\end{gathered}
$$

После введения обозначений

$$
\begin{aligned}
& C_{\mathrm{v} 12}=-C_{\mathrm{v} 11} A_{\mathrm{v} 1}, \\
& C_{\mathrm{v} 22}=-C_{\mathrm{v} 21} A_{\mathrm{v} 2},
\end{aligned}
$$

в результате решения системы уравнений (16.1) - (16.4) получено

$$
A_{\mathrm{v} 2}=-\frac{I_{1}\left(x_{\mathrm{v} 2} R_{4}\right)}{K_{1}\left(x_{\mathrm{v} 2} R_{4}\right)}
$$




$$
\begin{gathered}
A_{v 1}=-\frac{\frac{\mu_{R 1} \varkappa_{v 1}}{\mu_{R 2} \varkappa_{v 2}} \frac{I_{0}\left(\varkappa_{v 2} R_{2}\right)-A_{v 2} K_{0}\left(\varkappa_{v 2} R_{2}\right)}{I_{1}\left(\varkappa_{v 1} R_{2}\right)+A_{v 2} K_{1}\left(\varkappa_{v 2} R_{2}\right)} I_{1}\left(\varkappa_{v 1} R_{2}\right)-I_{0}\left(\varkappa_{v 1} R_{2}\right)}{\frac{\mu_{R 1} \varkappa_{v 1}}{\mu_{R 2} \varkappa_{v 2}} \cdot \frac{I_{0}\left(\varkappa_{v 2} R_{2}\right)-A_{v 2} K_{0}\left(\varkappa_{v 2} R_{2}\right)}{I_{1}\left(\varkappa_{v 2} R_{2}\right)+A_{v 2} K_{1}\left(\varkappa_{v 2} R_{2}\right)} K_{1}\left(\varkappa_{v 1} R_{2}\right)+K_{0}\left(\varkappa_{v 1} R_{2}\right)}, \\
C_{v 11}=-\frac{B_{\partial v}}{\mu_{R 1} \varkappa_{v 1}\left[I_{1}\left(\varkappa_{v 1} R_{1}\right)+A_{v 1} K_{1}\left(\varkappa_{v 1} R_{1}\right)\right]^{\prime}} \\
C_{v 21}=C_{v 11} \frac{I_{0}\left(\varkappa_{v 1} R_{2}\right)-A_{v 1} K_{0}\left(\varkappa_{v 1} R_{2}\right)}{I_{0}\left(\varkappa_{v 2} R_{2}\right)-A_{v 2} K_{0}\left(\varkappa_{v 2} R_{2}\right)} .
\end{gathered}
$$

Тем самым определены первые постоянные интегрирования; вторые находятся по выражениям (17.1) и (17.2).

При подстановке соответствующих величин в выражения (12) и (13) получаем для основной пространственной гармонической $v=1$ в первом слое

$$
\begin{gathered}
B_{R 11}=B_{01} \frac{I_{1}\left(\varkappa_{11} R\right)+A_{11} K_{1}\left(\varkappa_{11} R\right)}{I_{1}\left(\varkappa_{11} R_{1}\right)+A_{11} K_{1}\left(\varkappa_{11} R_{1}\right)} \cos \frac{\pi}{\tau_{1}} z, \\
H_{z 11}=-\frac{\pi}{\tau_{1}} \frac{B_{01}}{{ }^{\prime \prime} \varkappa_{11} \varkappa_{11}} \frac{\left[I_{0}\left(\varkappa_{11} R\right)-A_{11} K_{0}\left(\varkappa_{11} R\right)\right]}{\left[I_{1}\left(\varkappa_{11} R_{1}\right)+A_{11} K_{1}\left(\varkappa_{11} R_{1}\right)\right]} \sin \frac{\pi}{\tau_{1}} z .
\end{gathered}
$$

Магнитодвижущая сила, действующая между серединами соседних полюсов на поверхности статора при $v=1$, равна

$$
F_{p 1}=\int_{1 \frac{\pi}{\tau_{1}} z=\pi}^{1 \frac{z}{\tau_{1}} z=0}\left(H_{z 11}\right)_{R=R_{1}} d z=\frac{2 B_{\delta 1}}{\mu_{R 1} \varkappa_{11}} \frac{I_{0}\left(\varkappa_{11} R_{1}\right)-A_{11} K_{0}\left(\varkappa_{11} R_{1}\right)}{I_{1}\left(\varkappa_{11} R_{1}\right)+A_{11} K_{1}\left(\varkappa_{11} R_{1}\right)}
$$

Обозначим через $K_{F 1}$ отношение

$$
K_{F 1}=\frac{F_{1}}{F_{p 1}},
$$

где $F_{1}$ - амплитуда основной гармонической м.д.с. многофазной обмотки статора на пару полюсов. Она равна

$$
F_{1}=\frac{4 \sqrt{2}}{\pi 2} m \frac{I_{0} w_{1}}{p} k_{061} \frac{\sin \frac{\pi b}{\tau_{1} 2}}{\frac{\pi b}{\tau_{1} 2}}
$$

Здесь $m$ - число фаз; $I_{0}$ - ток намагничивания; $w_{1}$ - число витков фазы обмотки статора; $p$ - число пар полюсов; $k_{\text {об1 }}$ - обмоточный коэффициент для основной пространственной гармонической $v=1$; $b$ - ширина открытия паза. 
В обмотке статора основное магнитное поле наводит э.д.с.

$$
E_{1}=2 \sqrt{2} f w_{1} k_{061} B_{\text {ว่1 }} \tau_{1} l_{1}
$$

где $f$ - частота сети; $l_{1}$ - расчетная ширина пакета статора, равная

$$
l_{1}=2 \pi R_{1} \text {. }
$$

Индуктивное сопротивление обмотки статора, обусловленное основным магнитным полем, равно

$$
X_{m}=\frac{E_{1}}{I_{0}}
$$

При подстановке в выражение (29) необходимых величин на основании $(25),(26),(27)$ и (29) получается

$$
X_{m}=\frac{4}{\pi} \frac{m f w_{1}^{2} k_{\text {oó1 } 1}^{2} \tau_{1} l_{1} \quad \sin \frac{\pi b}{\tau_{1}^{2}}}{\frac{K_{F 1} p}{\mu_{R 1} \varkappa_{11}}\left[\frac{I_{0}\left(\varkappa_{11} R_{1}\right)-A_{11} K_{0}\left(\varkappa_{11} R_{1}\right)}{I_{1}\left(\varkappa_{11} R_{1}\right)+A_{11} K_{1}\left(\varkappa_{11} R_{1}\right)}\right] \frac{\pi b}{\tau_{1} 2}} .
$$

Аналогичным путем может быть определено любое индуктивное сопротивление обмотки статора $X_{m v}$, обусловленное $v$-ой пространственной гармонической магнитного потока.

При расчете асинхронных машин используется понятие об индуктивном сопротивлении $X_{g}$, обусловленном высшими пространственными гармоническими и представляющем собой следующую сумму:

$$
X_{k}=\underset{v \neq 1}{\Sigma} X_{m v}
$$

С учетом выражений для $X_{m v}$, аналогичных выражению (30), в случае цилиндрического насоса можно индуктивное сопротивление $X_{g}$ представить в виде

$$
X_{g}=X_{m} \sum_{v \neq 1} \frac{\frac{I_{0}\left(\varkappa_{11} R_{1}\right)-A_{11} K_{0}\left(\varkappa_{11} R_{1}\right)}{I_{1}\left(\varkappa_{11} R_{1}\right)+A_{11} K_{1}\left(\varkappa_{11} R_{1}\right)}}{\frac{I_{0}\left(\varkappa_{v 1} R_{1}\right)-A_{v 1} K_{0}\left(\varkappa_{v 1} R_{1}\right)}{I_{1}\left(\varkappa_{v 1} R_{1}\right)+A_{v 1} K_{1}\left(\varkappa_{\mathrm{v} 1} R_{1}\right)}} \cdot \frac{k_{06 v}^{2} \sin v \frac{\pi b}{\tau_{1} 2}}{v^{2} k_{061}^{2} \sin \frac{\pi b}{\tau_{1} 2}},
$$

где все обозначения совпадают с ранее принятыми.

\section{Л ИТЕРАТУРА}

1. А. И. Вольдек, Магнитное поле индукторов линейных электромагнитных насосов, Изв. Высших учебных заведеннй «Электромеханика», № 12, 1958.

Институт энергетики

Акадении наук Эстонской ССР
Поступила в редакцию 1. II 1961 


\section{LINEAARSETE SILINDRILISTE PUMPADE POHIMAGNEETIMISTAKISTUS}

\section{R. Parts}

\section{Resümee}

Artiklis vaadeldakse lineaarset silindrilist pumpa, mille kolmeíaasiline mähis asib välise staatoripaketi uuretes. Oldisel kujul lahendatakse magnetvälja jagunemine pumbakanalis ja sisemise staatori paketis, kusjuures eeldatakse, et pumbakanalis puuduvad voolud. Seega taandub ülesanne potentsiaalvörrandi lahendamisele. Seda tehakse üldisel kujul, vōttes arvesse erinevaid magnetilisi läbitavusi pumba aksiaal- ja radiaalsuunas. Seetōttu on saadud lahend, mis määrab magnetvälja jagunemise, rakendatav ka agregaatide arvutamiseks, mis on vajalikud ôhukeseseinaliste torude sisepindade töötlemiseks.

Lähtudes magnetvälja jagunemise üldlahendist, on tuntud vōtete abil leitud põhimagneetimistakistust $\left(X_{m}\right)$ ning kõrgemate ruumiliste harmooniliste induktiivtakistust $\left(X_{g}\right)$ määravad valemid, mis on kasutatavad nii paljupooluseliste silindriliste pumpade kui ka älalnimetatud torude sisepindade töötlemise agregaatide arvutamiseks.
Eesti NSV Teaduste Akadeemia Energeetika Instituut
Saabus toimetusse
1. II 1961

\section{THE MAIN INDUCTIVE RESISTANCE OF A RECTILINEAR CYLINDRICAL PUMP}

\section{R. Parts}

\section{Summary}

The object of the present study is a rectilinear cylindrical pump whose three-phase winding is placed in the slots of the exterior stator. First of all, the general distribution of the magnetic field in pump channel and in the inside stator is computed, proceeding from the condition that the pump channel is without a current. Thus the problem is limited to the solution of a differential equation of scalar potention. The solution is carried int in a general form, taking into account the different magnetic permeability in lengthwise and radial directions. In this way a determination of a magnetic field distribution has been obtained, which can also be applied for the calculation of the machine tools needed for the treatment of the inside-surfaces of small-wall tubes.

Proceeding from the general determination of the magnetic field, the main inductive resistance $\left(X_{m}\right)$ has been found, as well as formulas determining the inductive resistance conditioned by differential leakage $\left(X_{g}\right)$, which can be used for calculations of 2p-pole cylindrical pumps as well as of machine tools for the treatment of inside-surfaces of the aiorementioned tubes. Academy of Sciences of the Estonian S.S.R.,
Institute of Energetics
Received

February 1st, 1961 